\title{
Application of Thermal Neutron Activation for Autoradiographic Detection of Fingerprints
}

\author{
Hideo Isono and Hiroshi KozUKA \\ National Research Institute of Police Science, \\ 6, Sanban-cho, Chiyoda-ku, Tokyo, Japan \\ Received July 11, 1973
}

\section{Introduction}

Fingerprint detection is one of the useful methods for the crime investigation and is highly appreciated as an important method to prepare an absolute evidence even in the present time.

There have been many kinds of methods for the detection of prints of papillary lines. For example, aluminium powder method, ninhydrine method, silver nitrate method, iodine vapor method, fluorescein method, and benzidine method. If they are used for the suitable specimens, they will show their characteristic effects. It is not enough, however, to detect all of the fingerprints which are deposited on many kinds of materials only by these methods.

Recently, some kinds of radioisotopes are also used for the detection of fingerprints in some laboratory. After the paper specimens were treated with radioisotopic reagents conforming to the ordinal methods, the fingerprints are autoradiographically detected. In these methods, ${ }^{110 m} \mathrm{Ag}$ is used in the form of silver nitrate ${ }^{1,2)}$. ${ }^{131}$, in the form of iodine vapor. ${ }^{35} \mathrm{~S}$, in the form of sulfurous acid gas $^{3)}$. Natural uranium oxide, in the form of powder.

In the usual methods, chief mediums used for the fingerprint detection are chlorine, skin-fat, peptides, and amino acids in sweat. But, sodium has not been used for this purpose in spite of it's being the same chief component as the others. The method established in this paper can make use of sodium too.

\section{Experimental}

\subsection{Apparatus and Reagents}

Nuclear reactor: HTR (Tokyo Atomic Industrial Research Laboratory Co., Ltd.)

Power: $100 \mathrm{~kW}$

Thermal neutron flux: $2.1 \times 10^{12} n / \mathrm{cm}^{2} \cdot \mathrm{sec}$ (in the reactor core), $7.8 \times 10^{11} \mathrm{n} / \mathrm{cm}^{2} \cdot \mathrm{sec}$ (in the pneumatic tube or on the irradiation shelf)

Polyethylene capsule: $22 \mathrm{~mm} \phi \times 64 \mathrm{~mm}$ high (for the core), $18 \mathrm{~mm} \phi \times 64 \mathrm{~mm}$ high (for the pneumatic tube)

X-ray film: Fuji X-ray Film Industrial 200 (high contrast non-screen type) or 400 .

Developer: Fuji Developer Rendol(ED-R)

Fixature: Fuji Fix (FF-H)

\subsection{Specimens}

Paraffine paper (powder paper): used for wrapping the powder-drug, colorless, 0.025 $\mathrm{mm}$ thick, $10 \mathrm{~cm} \times 10 \mathrm{~cm}$, HAKUAI.

Thin letter paper: for copy, A4 type having ruled lines, colorless, $0.035 \mathrm{~mm}$ thick.

Paper: for ordinal notebook, having ruled lines, white, $0.1 \mathrm{~mm}$ thick, KOKUYO; for memorandum, having no line, white, $0.08 \mathrm{~mm}$ thick, $8 \mathrm{~cm} \times 10 \mathrm{~cm}$, KOKUYO.

Cellophane: used for covering a cigaret box, colorless, transparent, $0.025 \mathrm{~mm}$ thick.

Polyethylene sheet: colorless, transparent, $0.025 \mathrm{~mm}$ thick.

Label: paper, used for writing a name.

Government postcard: ordinal, $0.25 \mathrm{~mm}$ thick. 


\subsection{Method}

2.3.1 Irradiation in the core and in the pneumatic tube

Fingerprinted specimens (about $10 \mathrm{~cm} \times 10$ $\mathrm{cm}$ ) were sealed in a polyethylene bag. The sealed one was covered again like this with the other bag to be protected much more and was put into a polyethylene capsule without being crumpled up. After the irradiation in the reactor core or in the pneumatic tube, the specimens were stood still for a while and were directly contacted with a piece of X-ray film. If it is unknown on which side of a specimen fingerprints have been left, the one is sandwiched between two pieces of X-ray film. Development was carried out under the red-brown safe lamp (Fuji Safe Light Glass No. 5) observing the change of the film surface.

\subsubsection{Irradiation on the shelf}

The specimen twice covered with the polyethylene bags was fixed on the inside of a little arched lead plate and was stood on the irradiation shelf to be kept facing to the core. The other processes were the same as the before.

\section{$2 \cdot 4$ Results}

It was the first investigation whether activation autoradiography could be applied to the detection of the prints of papillary lines on a sheet. The specimens were prepared by only pressing the unwashed fingers onto the paraffin paper, thin letter paper, notebook's paper, memorandum-paper, label, government postcard, polyethylene sheet etc. They were irradiated for $20 \mathrm{~min}$ in the pneumatic tube with thermal neutron flux of 7.8 $\times 10^{11} n / \mathrm{cm}^{2} \cdot \mathrm{sec}$. After the decay of short half life nuclides by leaving the specimens alone for $2 \mathrm{hr}$ (cooling time), they were directly contacted onto the X-ray film for 15 hr (exposure time).

As a result of the first trial, it was found that only the prints of papillary lines on the paraffin paper, the thin letter paper, and the polyethylene sheet could be clearly detected. The background of the film was so transparent that something could be seen very clearly through itself. But the whole of the film was made so black by the others that any autoradiograms could not been shown. The darkness of the background could not been improved even by changing irradiation time and cooling time: $[45 \mathrm{~min}$ and $60 \mathrm{~min}$ ], [30 $\mathrm{min}$ and $30 \mathrm{~min}$, and [20 min and $40 \mathrm{~min}$ ] respectively in each. The paraffin paper and the thin letter paper had shown good results under the following conditions: the thermal neutron flux of 7.8 $\times 10^{11} \mathrm{n} / \mathrm{cm}^{2} \cdot \mathrm{sec}$, the irradiation time of 30 min, the cooling time of $30 \mathrm{~min}$, the exposure time of $17 \mathrm{hr}$. They were successively exposed again onto the X-ray film for $24 \mathrm{hr}$ with the total cooling time of $17 \mathrm{hr}$. As a result of the second exposure, it was found that the fingerprints on these specimens could be detected very clearly even after the long cooling time.

In order to know the effects of high neutron flux and long irradiation time, the fingerprinted paraffin paper was irradiated for $5 \mathrm{hr}$ at the thermal neutron flux of 2.1 $\times 10^{12} n / \mathrm{cm}^{2} \cdot \mathrm{sec}$, and was exposed to the $\mathrm{X}$ ray film for $5 \mathrm{hr}$ with the cooling time of $4 \mathrm{hr}$. As a result of it, clear autoradiograms could be obtained. The same specimen was contacted again onto the X-ray film for 12 hr with the total cooling time of $10 \mathrm{hr}$. The second trial could show good result.

The next, this method was applied to the actual samples. As the samples, nineteen sheets of paraffin paper which had been used for wrapping up powdered drug were collected and were irradiated for $5 \mathrm{hr}$ at the thermal neutron flux of $2.1 \times 10^{12} n / \mathrm{cm}^{2} \cdot \mathrm{sec}$.

First of all, eight of the samples were exposed to the X-ray film for $4 \mathrm{hr}$ after the cooling of $6 \mathrm{hr}$. Good results were obtained in every case. After the first trial, they were tested again under the next condition: the exposure time of $10 \mathrm{hr}$ and the total cooling time of $12 \mathrm{hr}$. From every sample, the autoradiographic fingerprints could be shown clearly on the X-ray film and appeared at the position where the fingers should have been touched at the time of wrapping.

In order to know the effect of the cool- 
ing time and the exposure time, these time conditions were varyed. a) The number of actual samples $(\mathrm{N})=6$, the first exposure: $\mathrm{CT}=6 \mathrm{hr}, \mathrm{ET}=11 \mathrm{hr}$, the second exposure (the same samples were exposed again to the X-ray film): $\mathrm{CT}=24 \mathrm{hr}, \mathrm{ET}=42 \mathrm{hr}, \mathrm{b}) \mathrm{N}=$ 2 , the first exposure: $\mathrm{CT}=36 \mathrm{hr}, \mathrm{ET}=10 \mathrm{hr}$, c) $\mathrm{N}=3$, the first exposure: $\mathrm{CT}=22 \mathrm{hr}, \mathrm{ET}=$ $48 \mathrm{hr}$. Under these conditions, every actual sample showed good result.

\section{Discussion}

The principle of activation autoradiography is much depend on the fact that a material deposited on a sample at the touch of finger contains such elements that can be activated with thermal neutron. So, the deposited material itself is not problem on this method.

First of all, as one of the materials, sweat must be descrived. It has many kinds of components to be detected by the ordinal fingerprint detection method. Sodium and chlorine which are main components in sweat can be sensitively activated with the thermal neutron.

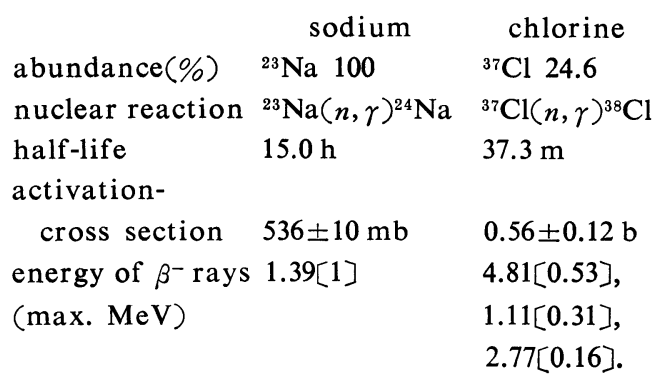

energy of $\gamma$ rays $1.37[1], 2.75[1] .1 .60(100)$,

$2.15(130)$

[ ]: ratio per decay.

( ): relative intensity.

Chlorine has been already used for silver nitrate method. But, there has not been any chemical or physical fingerprint detection method to make use of sodium.

Not only chlorine but also sodium is very useful for this method and more it is possible to expose the long irradiated sample to the X-ray film after a long cooling time because the half-life of ${ }^{24} \mathrm{Na}$ is much longer than that of ${ }^{38} \mathrm{Cl}$.
If this method is applied to the paraffin paper, the thin letter paper, or the polyethylene sheet, it will demonstrate its ability. But, it is very difficult to be applied to such specimens containing much activable element as the cellophane, the paper for a notebook or for a label, and the government postcard. It is possible to try the ordinal fingerprint detection method to the irradiated sample because the state of the sample-surface is not disturbed with this method.

Through the processes of this method, it is the most important point that the activated sample must be contacted to the X-ray film as closely as possible. It is much dependent on this point whether the clean autoradiograms of the papillary lines could be obtained or not. From this point, the samples must be treated not to be crumpled up, because it is very difficult for the crumpled parts of them to be closely contacted to the X-ray film only by pressing them in the $X$-ray film case.

\section{Conclusions}

In this paper, the experiments were carried out under the following conditions.

A: Neutron flux $7.8 \times 10^{11} n / \mathrm{cm}^{2} \cdot \mathrm{sec}$ Irradiation time $20-45 \mathrm{~min}$ Cooling time $30-120 \mathrm{~min}$ Exposure time $15-20 \mathrm{hr}$

B: Neutron flux $2.1 \times 10^{12} n / \mathrm{cm}^{2} \cdot \mathrm{sec}$ Irradiation time $5 \mathrm{hr}$ Cooling time 4-6 hr [or 10-24 hr] Exposure time 5-11 hr [or 10-48 hr]

Under these conditions, it was found that clear autoradiograms of papillary lines could be obtained from the specimens of paraffin paper, thin letter paper, and polyethylene-sheet.

There are some merits in this application of neutron activation autoradiography to the fingerprints-detection.

1) Sodium which is one of the main components in sweat is very easily detected by thermal neutron activation, while it cannot be detected by any chemical reaction.

2) This method can be applied to such a thin specimens as paraffin paper, thin 


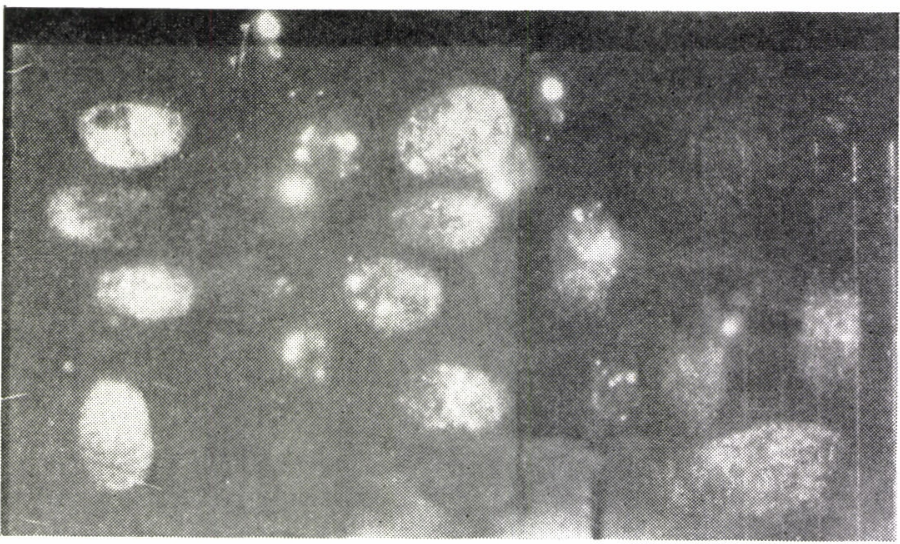

Photo 1 Test samples. Left: Paraffin paper. Right: Thin letter paper. Thermal neutron flux: $3.7 \times 10^{11} \mathrm{n} / \mathrm{cm}^{2} \cdot \mathrm{sec}$. Irradiation time: 30 min. Cooling time: $30 \mathrm{~min}(18 \mathrm{~h})$. Exposure time: $17 \mathrm{~h}(24 \mathrm{~h})$. After the first exposuring, the same sample was tried again under the conditions shown in the parentheses.

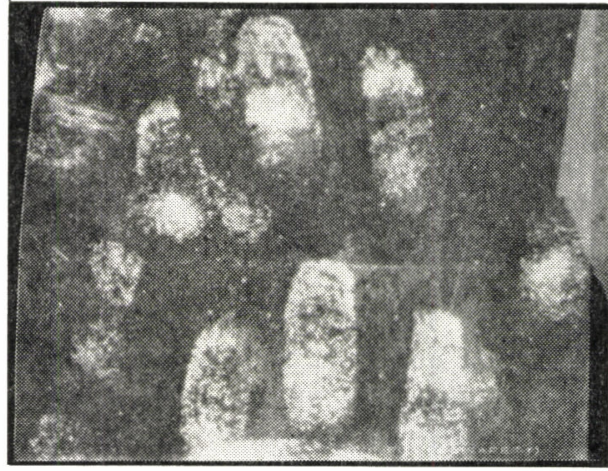

Photo 2 Test sample: Paraffin paper. Thermal neutron flux: $2.1 \times 10^{12}$ $n / \mathrm{cm}^{2} \cdot \mathrm{sec}$. Irradiation time: $5 \mathrm{~h}$. Cooling time: 4h. Exposure time: $5 \mathrm{~h}$.

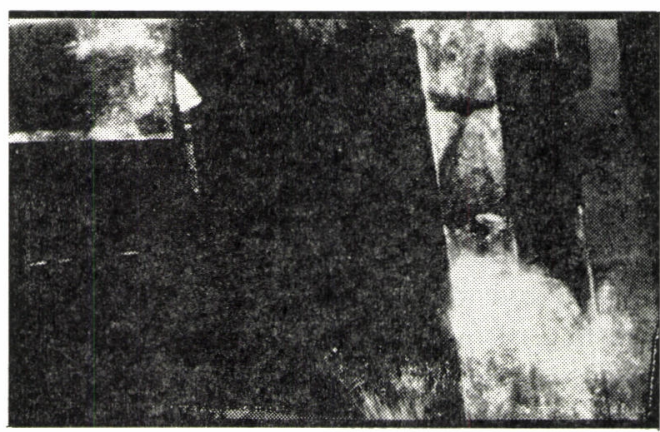

Photo 4 Actual sample: Paraffine paper. Thermal neutron flux: $2.1 \times 10^{12} n$ / $\mathrm{cm}^{2} \cdot \mathrm{sec}$. Irradiation time: $5 \mathrm{~h}$. Cooling time: 22h. Exposure time: 48 h.

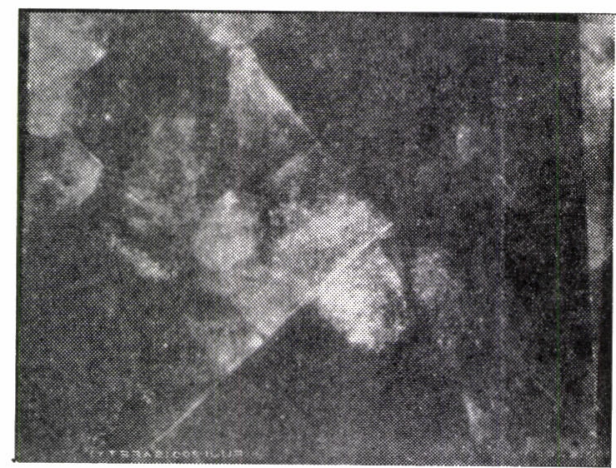

Photo 3 Actual sample: Paraffin paper. Thermal neutron flux: $2.1 \times 10^{12}$ $n / \mathrm{cm}^{2} \cdot \mathrm{sec}$. Irradiation time: $5 \mathrm{~h}$. Cooling time: $6 \mathrm{~h}$. Exposure time: $4 \mathrm{~h}$.

letter paper, or polyethylene-sheet, on which papillary lines are often disturbed by wet or powder method for fingerprints-detection as a results of capillary or electrostatic effect.

\section{References}

1) L. Lelental and A. Szuchnik: Intern. J. Appl. Radiat. Isotopes, 15, 373 (1964)

2) K. Prokopowicz, K. Akerman, and A. Szuchnik: ibid., 17, 657 (1966)

3) L. Grantr, F.L. Hudson, and J.A. Hockey: Paper Maker, 149, 47 (1965) 